\title{
On the Performance of All Optical Wireless Communications Based on Optical Nonlinear Generators
}

\author{
Lwaa Faisal Abdulameer ${ }^{1}$, Ali Salah Mahdi ${ }^{2}$ \\ ${ }^{1,2}$ University of BaghdadAl-Khwarizmi, College of Engineering. \\ lwaa@kecbu.uobaghdad.edu.iq
}

\begin{abstract}
Erbium Doped Fiber Ring Laser (EDFRL) and MachZehnder Interferometer (MZI) are being used to generate optical nonlinear signals to increase the level of security. Using nonlinear components in communication systems are the leading cause of inferiority in all system performance. One of the most significant current adopted to improve system performance is to use multiple sources at transmitter and multiple sources at receiver. The aim of this paper is to explore this technology to reduce the overall system complexity and improve its performance via designing an all optical wireless diversity technique to benefit from generating the nonlinear optical signals by the optical components. The design of different optical nonlinear modulation schemes for optical wireless communications is presented in this paper. EDFRL and MZI are important components in optical systems used to generate nonlinear optical for secure communications which are generate sequence by exploiting nonlinearities. We evaluate and compare the Bit-Error Rate (BER) versus SNR at a distance of 500 meter between optical emitter and detector in a wireless channel using electrical generators and optical generators (all optical components). We compared the BER performance for different diversity schemes whereas the results show the performance superiority for the system with multiple inputs (LEDs) and multiple outputs (photodetectors) as compared with systems using single input single output. We used OPTSYS software to simulate the results.
\end{abstract}

Keywords: optical, EDFRL, MZI.

Paper History :(received: 28-11-2019 ; Accepted: 12-12020)

\section{Introduction}

Recently, researchers have shown an increased interest in free space optics because of it offers high directional beams and high spectral effiecincy [1,2]. Optical wireless communication systems are designed by sending a laser beam through the free space channel between couple of Line-of-Sight points. Although relatively the distance between LOS points is unaffected by the troposphere conditions such as rain and snow when the distance is a few hundred meters, inhomogeneties in the pressure and temperature of the atmosphere can decline the received signal quality at the receiver $[3,4]$. It is well established that fading which caused by atmospheric turbulence can impair the overall system performance. The significantly technique to improve the quality of the received power is to deploy multiple lasers at the emitter and multiple detectors at the receiver [5-9]. In this paper, nonlinear sequences are generated using nonlinear electrical generator, Mach-Zehnder Interferometer (MZI) and Erbium Doped Fiber Ring Laser (EDFRL). MZI and EDFRL are ideal candidate for emitter of optical systems under the various operating conditions, they are already nonlinear devices that exhibit nonlinear dynamical behavior. Nonlinear signals which are generated by the electrical components have a features are similar to signals which are utlized in broad-spectrum communications where bearing signals are hidden within the noisy signals. For the present, the relatively simple electrical circuits have been generally used as methods for generating nonlinear sequences, but these approaches have two main problems. First, the nonlinear carrier sequences generated electronically have low level of privacy when applied to secure communication transmission. Second, the audio range in the most circuits designed is in the range of tens of $\mathrm{KHz}$ message bandwidths. Although, Radio frequency circuits might be complicated, it is difficult to gain some $\mathrm{GHz}$ frequencies desired in wideband channels. Moreever, most preveously introduced, high speed networks depend on optical fiber and free space optics. In this way, a considrable scheme based on an optical components is extremely eligible. Both of these issues are overcome by using nonlinear optical generators components [10,11]. Communications based on nonlinear generators have revolted a lot of interest. The main properties of these nonlinear signals are behaves like broadband signals and they are very sensitive dependence on initial conditions [12-14]. In this paper, the design of nonlinear MZI and EDFRL optical generators based on loss modulation are achieved.

\section{Optical nonlinear generator}

\section{A. Mach-Zehnder Interferometer (MZI) [10, 13-14]}

MZI nonlinear generator serves as an encoder for optical wireless channel based on non-periodic signals. For Chebyshev map, the Chebyshev polynomials $T_{m}(x), q \geq$ 2 are defined

$T_{m}(\cos \theta)=\cos (q \theta)$, where

$T_{2}(x)=2 x^{2}-1, T_{3}(x)=4 x^{3}-3 x, \ldots$. 
The Chebyshev map can be represented as:

$x_{n+1}=T_{q}\left(x_{n}\right), \quad x_{n} \in[-1,1]$

Where $x_{n}$ and $x_{n+1}$ represet the current and next states of the nonlinear sequences.

From equation (1), we can obtain

$x_{n}=\cos (\theta), x_{n+1}=\cos (q \theta)$

The modulation of electric field is shown in Fig (1).. However, the input electric field is

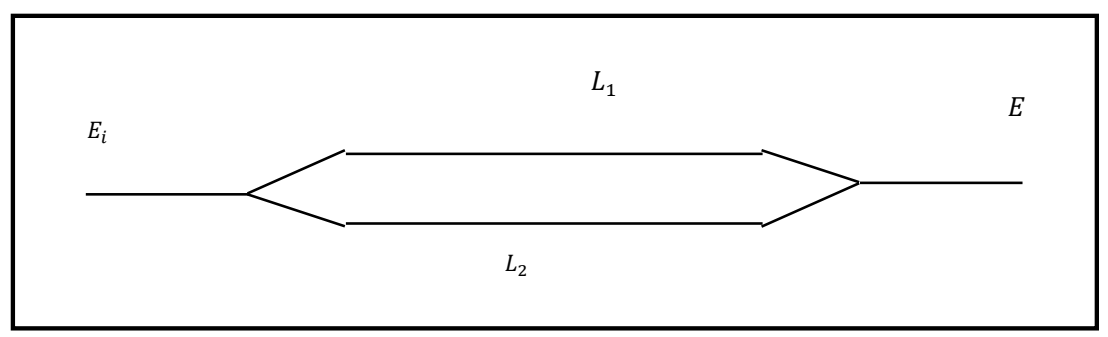

Fig (1) Interferometer of the Mach-Zehnder

The beam of the emitted light splits into two branches after the first $\mathrm{dB}$ coupler, where each bath has equal power

$P_{i 1}=P_{i 2}=A^{2} / 2$

The electron field can be individually shown as

$E_{1}=\frac{A}{\sqrt{2}} e^{j w\left(t-\frac{L_{1}}{v i_{1}}\right)}$

$E_{2}=\frac{A}{\sqrt{2}} e^{j w\left(t-\frac{L_{2}}{v i_{2}}\right)}$

However, the two branches have the same polarization after the path delays. Where $v$ represents the speed of light in free space. Moreover, the recombined electric field at the output of MZI is

$E=E_{1}+E_{2}$

$E=\frac{A}{\sqrt{2}}\left[e^{j w\left(t-L_{1} / v i_{1}\right.}+e^{j w\left(t-L_{2} / v i_{2}\right.}\right]=\frac{A}{\sqrt{2}} e^{j w t}\left[\cos \frac{w L_{1}}{v}+\right.$ $\left.\cos \frac{w L_{2}}{v}-i\left(\sin \frac{w L_{1}}{v}+\sin \frac{w L_{2}}{v}\right)\right] i_{1}=$

$\sqrt{2} \cos \left(\frac{\frac{w L_{1}}{v}+\frac{w L_{2}}{v}}{2}\right) e^{-j\left(\frac{w L_{1}}{v}+\frac{w L_{2}}{v}\right) / 2} A e^{j w t} i_{1}$

$$
E_{i}=A e^{j w t} i_{l}
$$

Where $A, w$ and $i_{l}$ in equation (4) represent the amplitude, the frequency and the polarization respectively. Equation (5) represents the input power,

$$
P=E_{i} . E_{i}^{*}=A^{2}
$$

Let $e^{-j\left(\frac{w L_{1}}{v}+\frac{w L_{2}}{v}\right) / 2}=1$, the phase shift $\frac{w L_{1}}{v}$ and $\frac{w L_{2}}{v}$ intrduced by the two arms satisfy

$\frac{w L_{1}}{v}+\frac{w L_{2}}{v}=2 k \pi$, thus $L_{I 1}+L_{i 2}=\frac{k \lambda}{n}$

$\frac{w L_{(i+1) 1}}{v}=q \frac{w L_{i}}{v}$, thus $L_{(i+1)}=q L_{i 1}$

Where $L_{i 1}$ and $L_{i 2}$ represent the length of the arm of the ith interferometer, $n$ is the refrective index and $\lambda$ is the wavelength. It is familiar that the electron fields $E_{1}, E_{2}, \ldots \ldots E_{N}$ output from each MZI are the product of the input electic field and the nonlinear sequences satisfying the Chebyshev map. Due to the important characterstics for the nonlinear components, any small change in initial conditions i.e., the wavelength $\lambda$ as well as the arm lengths of the interferometers $L_{i 1}$ and $L_{i 2}$ in terms of equations (11) and (12), the new nonlinear sequence will be obtaind

$L_{i+1}=\cos \left(2 \pi \cdot q \cdot n \cdot \frac{L_{i}}{\lambda}\right)$

As shown in Fig. (2), for each branch, a fiber optic delay line emitts the pulse to the suitable position, and the sum of the phase shift intrduced by the fiber optic delay line and the phase shift is zero. 
$L_{11}$

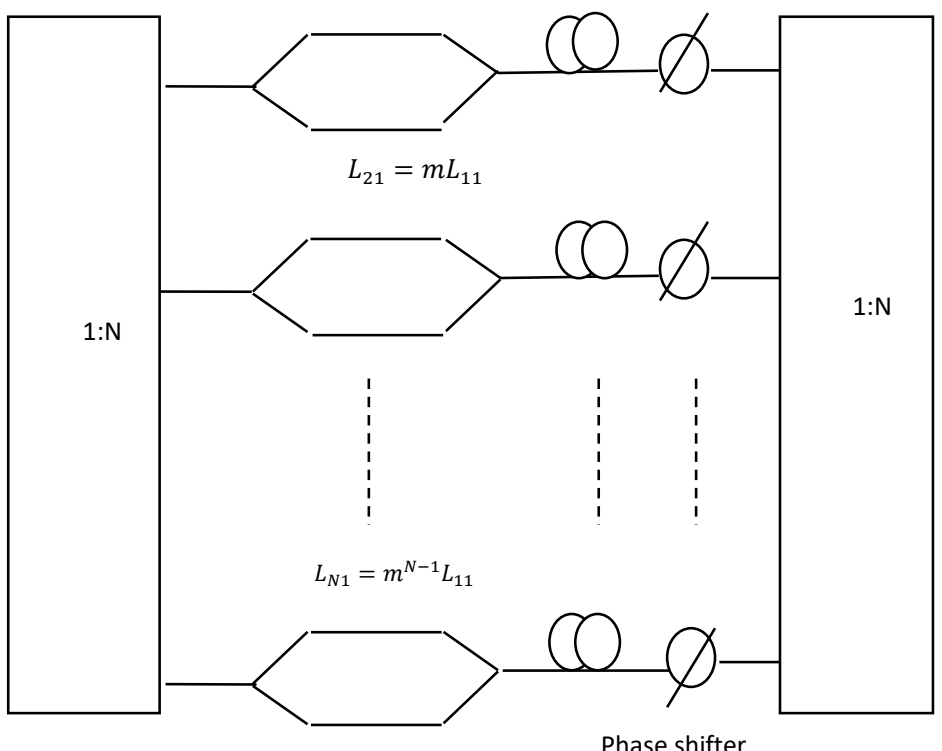

Fig. (2) Schematic of nonlinear sequence generator composed of MZI, phase shifter and fiber optic delay line [10] While Fig. (3)Shows the nonlinear sequences produced by MZI

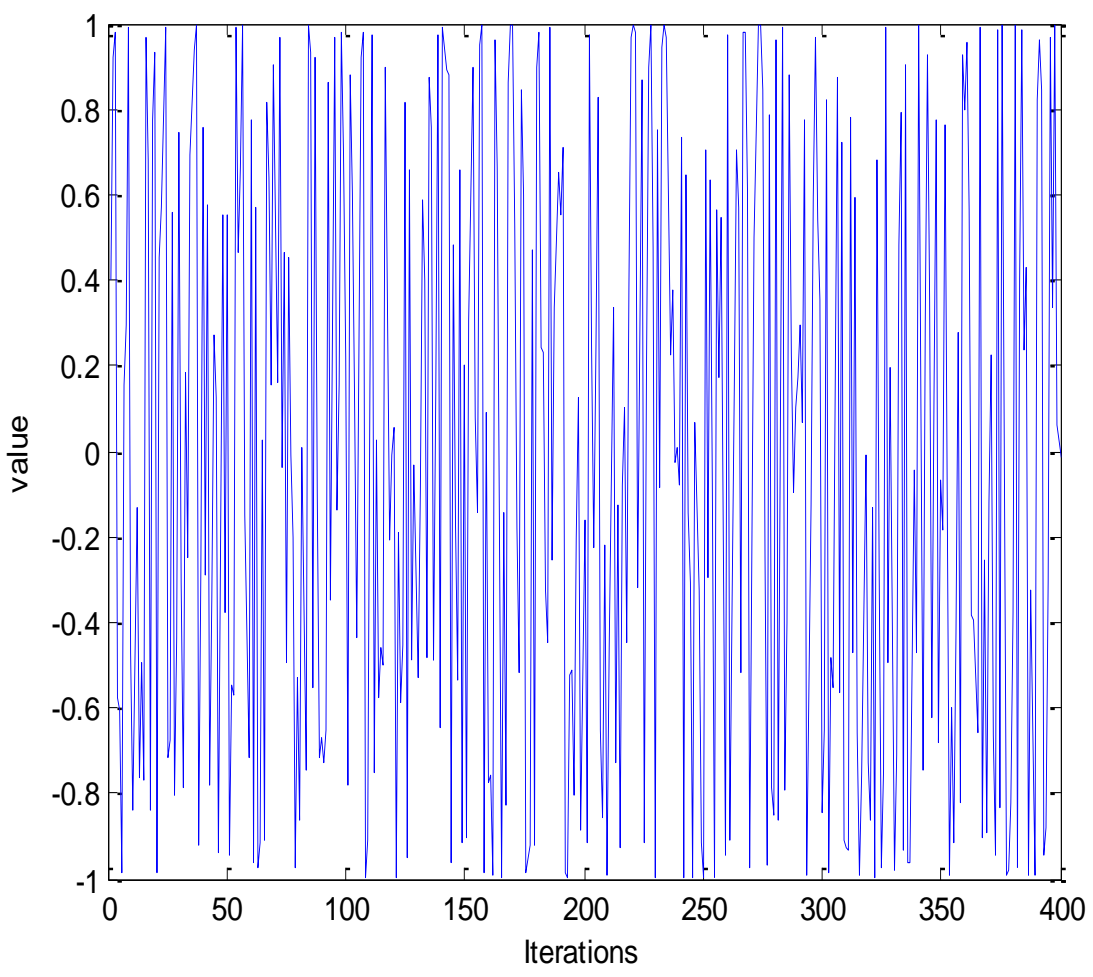

Fig. (3) Nonlinear sequence generation by MZI. 
Fig (4) Shows the design of Single Input Single Output (SISO) MZI transmitter and receiver system linked via the channel modeled as Gamma-Gamma distribution.

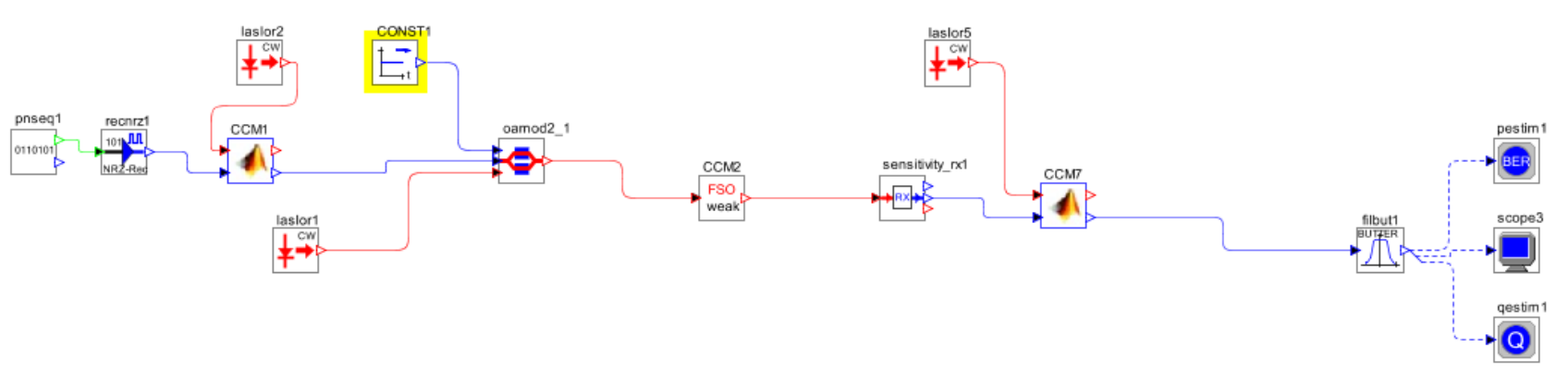

Fig (4) Proposed SISO MZI system architecture

Here, MZI modulator block is used instead of linear amplitude modulator block. The MATLAB blocks CCM1 in Fig.(4) is the nonlinear modulator. It picks up a raw data as input and produce non-periodic sequences at output using optical nonlinear generator designed by MZI. In a similar way, CCM7 is the nonlinear demodulator which picks up received signal as input and produces demodulated data as output.

Fig. (5)Shows the results obtained in the form of Electrical Spectrum of the SISO MZI. It is observed at the receiving end.

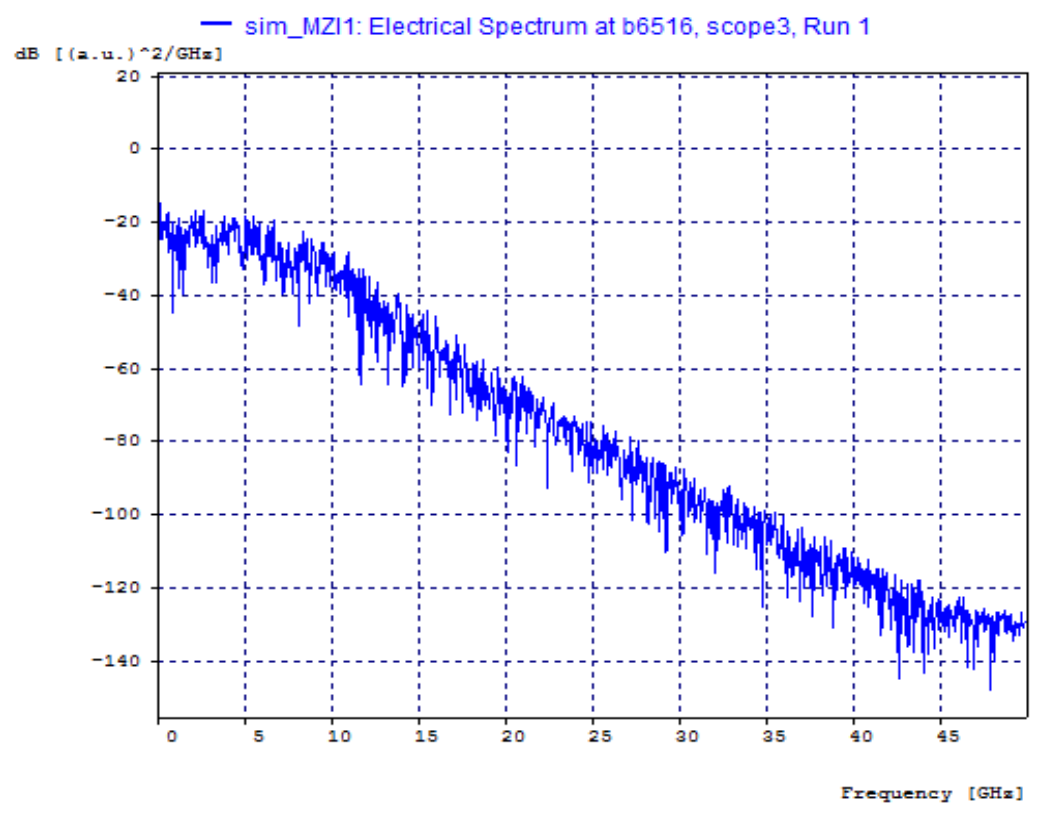

Fig. (5) Electrical Spectrum of SISO MZI

B. Erbium Doped Fiber Ring Laser (EDFRL) [15-17]

EDFRLs are used to generate optical nonlinear sequences by using loss modulation through variation of driving conditions and cavity parameters. Fig.(6)Shows the generation of optical nonlinear signals through EDFRL using electro-optical modulator for cavity loss modulation in the fiber loop. 


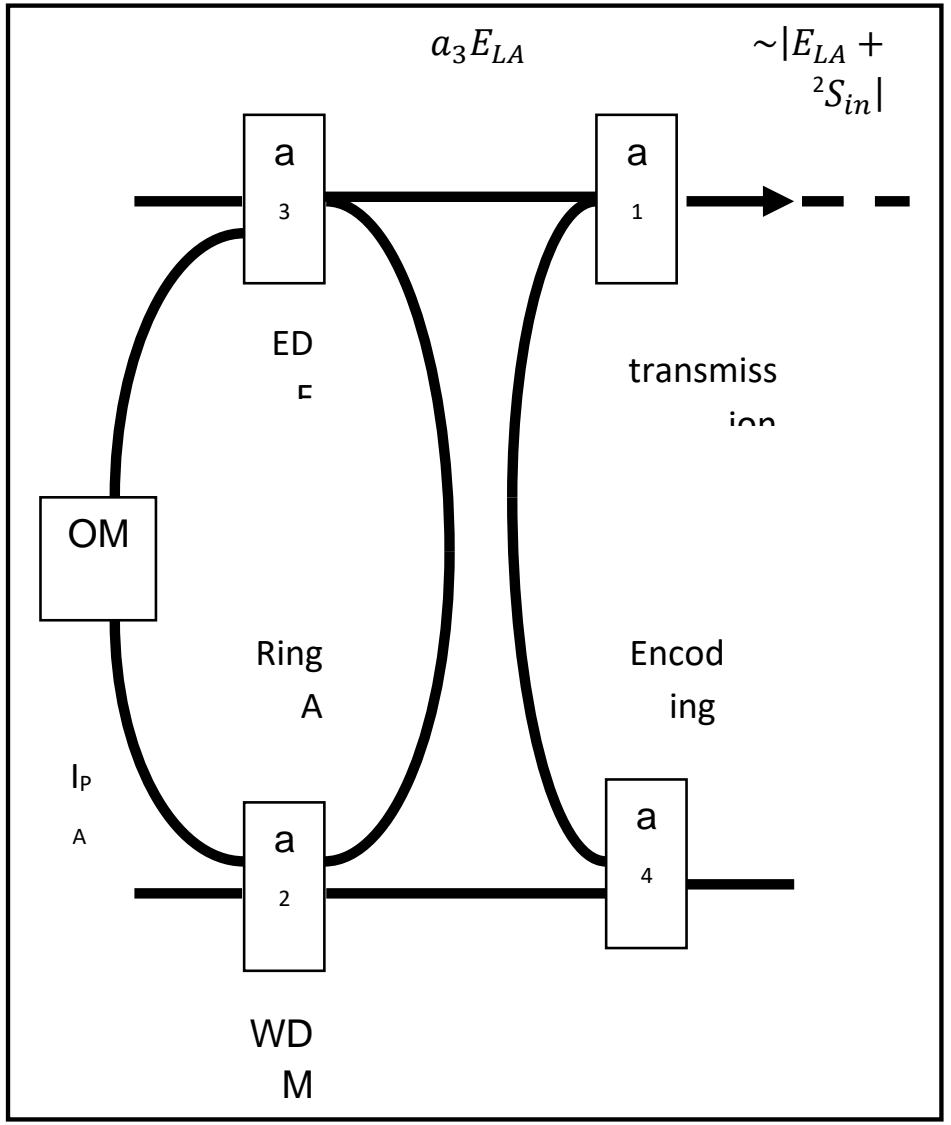

Fig (6) EDFRL transmitter ring [17].

The mathematical model to describe EDFRL given by laser rate equation as follows,

$$
\begin{aligned}
& \dot{E_{L A}^{*}}=-k_{a}\left(E_{L A}-c_{a} S_{i n}\right)+g_{a} E_{L A} D_{A}+\xi_{L A} \\
& \dot{D_{A}}=-\frac{1}{\tau}\left[\left(1+I_{P A}+E_{L A}^{2}\right) D_{A}-I_{P A}+1\right] \\
& k_{a}=k_{a 0}\left(1+m_{a} \sin \left(w_{a} t\right)\right)
\end{aligned}
$$

$$
S_{\text {in }}=S_{0}\left(1-\sin \left(w_{s} t\right)\right)
$$

Where $E_{L A}$ represents the lasing field, $D_{A}$ is population inversion density.

Table1 presents the parameters used in the current work, symbols, practical range and the optimum values to drive the system into nonlinear termed as reference values.

TABLE I: PARAMETERS of EDFRL [15].

\begin{tabular}{|c|c|c|c|}
\hline Parameter & symbol & reference & Practical range \\
\hline Decay Time Meta-Stable State & $\tau$ & $10 \mathrm{~m} \mathrm{sec}$ & Fixed \\
\hline Spontaneous Emission Factor & $\xi_{L A}$ & $10^{-4}$ & Fixed \\
\hline Pump power & $I_{P A}$ & $10 \mathrm{~m} \mathrm{~W}$ & $10 \mathrm{~mW}-200 \mathrm{~m} \mathrm{~W}$ \\
\hline Modulation Index & $m_{a}$ & 0.03 & $0.01-1$ \\
\hline Decay Rate & $k_{a 0}$ & $3.3 \times 10^{7}$ & $1 \times 10^{7}-5 \times 10^{7}$ \\
\hline Gain & $g_{a}$ & $2 k_{a 0}$ & $1.2 k_{a 0}-2 k_{a 0}$ \\
\hline Message Amplitude & $S_{0}$ & 1 & $0.1-10$ \\
\hline Modulating Frequency & $w_{a}$ & $3.5 \times 10^{5}$ & $3.5 \times 10^{5}-35 \times 10^{5}$ \\
\hline Message Frequency & $w_{S}$ & $3.14 \times 10^{5}$ & $3.14 \times 10^{5}-31.4 \times 10^{5}$ \\
\hline
\end{tabular}

Equations (14) to (17) are resolved using fourth order Runge-Kutta method as given in the following equations

$$
\begin{aligned}
& E_{L A(n+1)}=E_{L A(n)}+\frac{1}{6}\left(a_{E}+2 b_{E}+2 c_{E}+d_{E}\right) \\
& D_{A(n+1)}=D_{A(n)}+\frac{1}{6}\left(a_{D}+2 b_{D}+2 c_{D}+d_{D}\right)
\end{aligned}
$$




$$
\begin{aligned}
& a_{E}=-k_{a}\left(E_{L A(n)}-c_{a} S_{i n}\right)+g_{a} E_{L A(n)} D_{A(n)}+\xi_{L A} \\
& a_{D}=-\frac{1}{\tau}\left[\left(1+I_{P A}+E_{L A}^{2}\right) D_{A(n)}-I_{P A}+1\right] \\
& \left.b_{E}=-k_{a}\left(\left(E_{L A(n)}+\frac{a_{E}}{2}\right)-c_{a} S_{i n}\right)+g_{a} E_{L A(n)}+\frac{a_{E}}{2}\right)
\end{aligned}
$$

$$
\begin{aligned}
& b_{D}=-\frac{1}{\tau}\left[\left(1+I_{P A}+\left(E_{L A(n)}+\frac{a_{E}}{2}\right)^{2}\right)\left(D_{A(n)}+\frac{a_{D}}{2}\right)-\right. \\
& I_{P A}+1
\end{aligned}
$$

$c_{E}=-k_{a}\left(\left(E_{L A(n)}+\frac{b_{E}}{2}\right)-c_{a} S_{i n}\right)+g_{a}\left(E_{L A(n)}+\right.$

$\left.\frac{b_{E}}{2}\right)\left(D_{A(n)}+\frac{b_{D}}{2}\right)+\xi_{L A}$

$c_{D}=-\frac{1}{\tau}\left[\left(1+I_{P A}+\left(E_{L A(n)}+\frac{b_{E}}{2}\right)^{2}\left(D_{A(n)}+\frac{b_{D}}{2}\right)-\right.\right.$ $\left.I_{P A}+1\right]$
$d_{E}=-k_{a}\left(\left(E_{L A(n)}+c_{E}\right)-c_{a} S_{i n}\right)+g_{a}\left(E_{L A(n)}+\right.$ $\left.c_{E}\right)\left(D_{A(n)}+c_{D}\right)+\xi_{L A}$

(26)

$d_{D}=-\frac{1}{\tau}\left[\left(1+I_{P A}+\left(E_{L A(n)}+c_{E}\right)^{2}\right)\left(D_{A(n)}+c_{D}\right)-\right.$ $I_{P A}+1$

Equations above clears that the EDFRL is a second order and coupled system. The system dynamic ruled by two rate equations one for field $E_{L A}$ and other for population inversion $D_{A}$ as given by equations (14) to (17).

Fig.(7)Shows the nonlinear sequences generated by EDFRL for the parameters defined in table (1).

While Fig.(8)Shows the design of Single Input Single Output (SISO) EDFRL transmitter and receiver system linked via the channel modeled as Gamma-Gamma distribution.

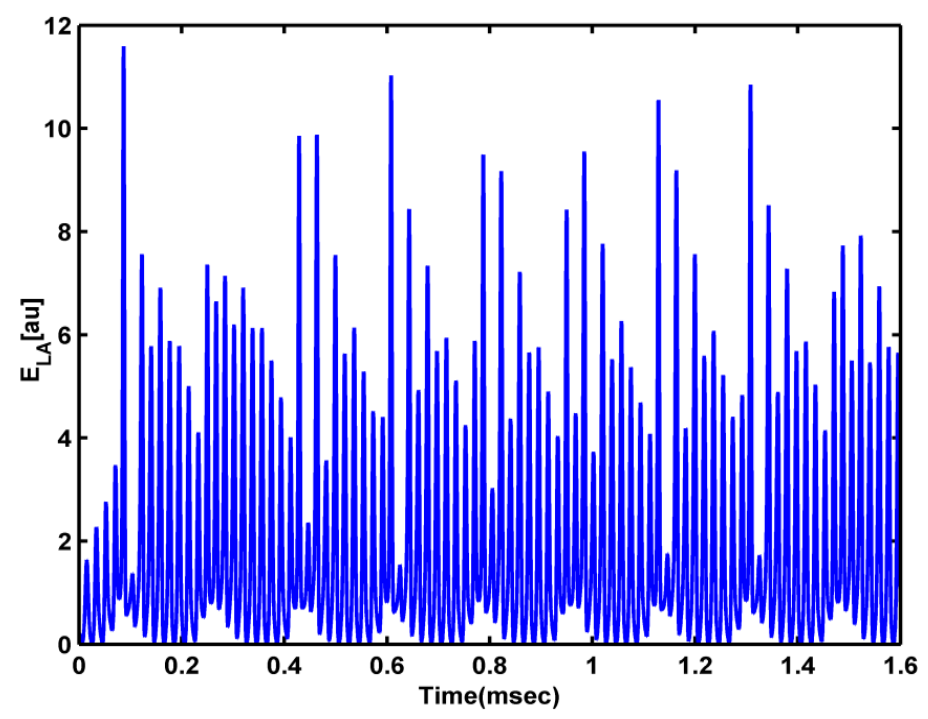

Fig (7) Generation of nonlinear sequence for reference model values

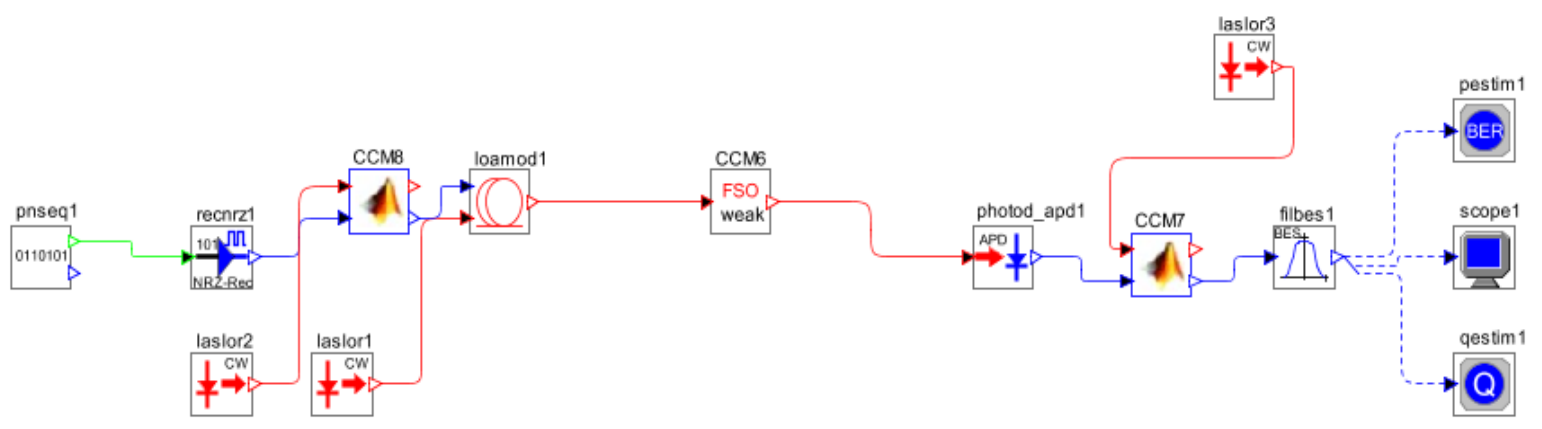

Fig (8) Proposed SISO EDFRL system architecture 


\section{Simulation Results and Discussions}

Prior to illustrating the results, the Bit Error Rate (BER) for the $2 \times 2$ free space optics used to determine the system performance can be expressed in equation (28)

$$
B E R=\sum_{l=1}^{m} \frac{1}{2} \operatorname{erfc}\left(\sqrt{\left[\frac{2 P_{T}^{2} R\left(h_{11}^{2}+h_{21}^{2}+h_{12}^{2}+h_{22}^{2}\right)}{Z}\right)}\right.
$$

Where $h_{i j}$ is the channel gain of the fading channel, $P_{T}$ is the radiated power transmitted by the LED, $R$ is the responsivity of the photodetector, $Z$ represents the noise power and mrepresents the number of points in the histogram.

Whereas, the BER for the $2 \times 2$ channel computed for the systems treated electrically can be expressed as in equation (29),

$$
B E R=\sum_{l=1}^{m} \frac{1}{2} \operatorname{erf} c\left(\sqrt{\frac{\left(h_{11}^{2}+h_{12}^{2}+h_{21}^{2}+h_{22}^{2}\right) E_{b}}{Z}}\right)
$$

Where $E_{b}$ is the energy of each bit in nonlinear sequence [18].

We have simulated results for two methods of nonlinear generators which are MZI nonlinear modulation and EDFRL nonlinear modulation. The results are simulated using the OPTSYS software package. The design uses MATLAB co-simulation for nonlinear sequences generation. Figures below shows the BER performance of different diversity techniques compared at $10^{-5}$ for systems with electrical circuit (Figures 9,10 and 11) and systems using all optical components (figures 12 and 13). It can be observed that $2 \times 1$ Fig. (11) gains $1 \mathrm{~dB}$ as compared to SISO Fig.(10). $2 \times 2$ gives $1.75 \mathrm{~dB}$ gain as compared to SISO. MZI-SISO Fig.(12) Gives BER performance equivalent to SISO Fig.(9) But with reduce complexity due to use all optical components.MZI system is all optical communication system, thus providing with less complex system. EDFRL (Fig. 13) also helps in reducing complexity since EDFRL-SISO gives performance equivalent to $2 \times 2$ (Fig. 11).

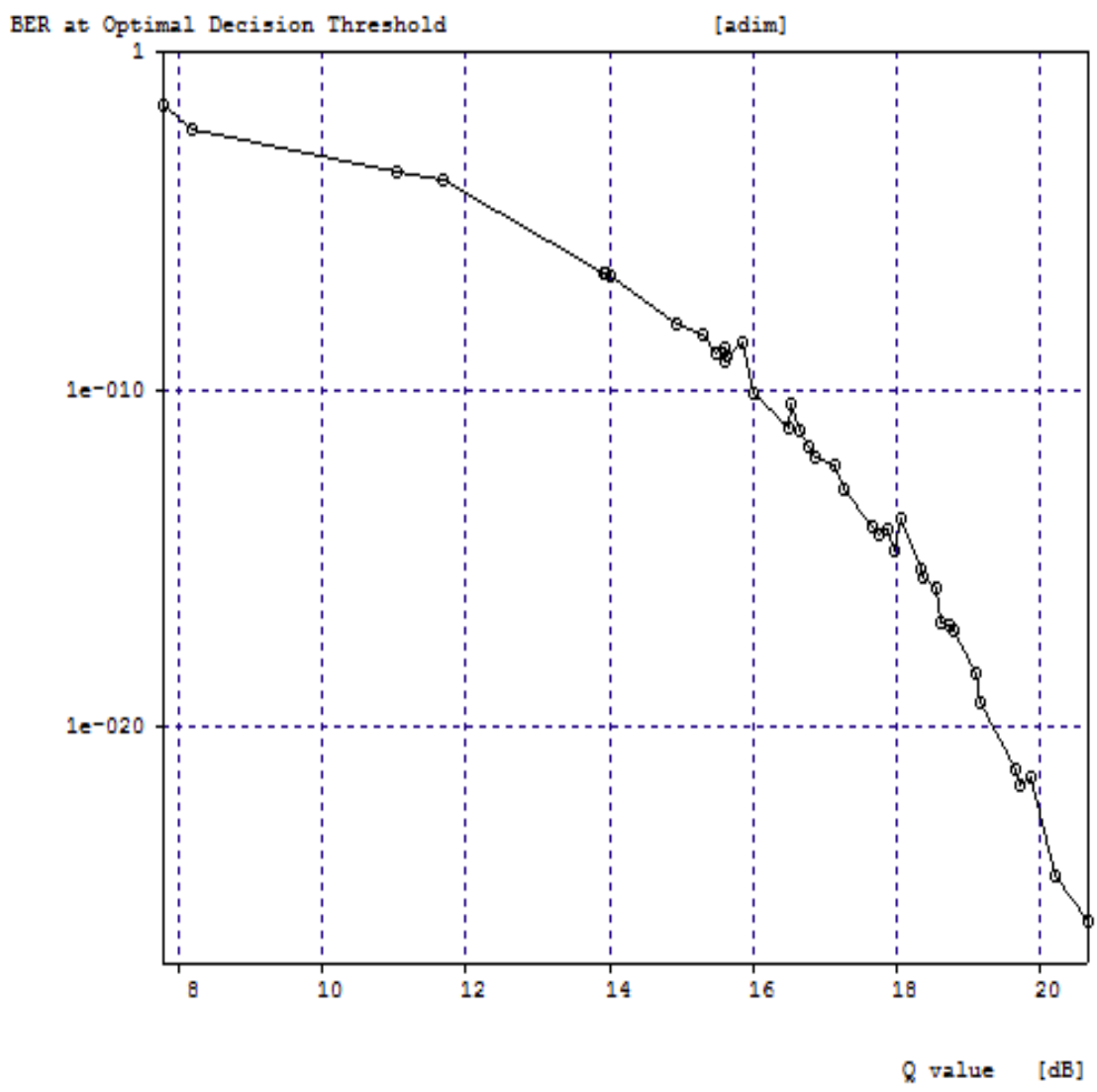

Fig (9) Computed BER with electrical generator for SISO communication system 


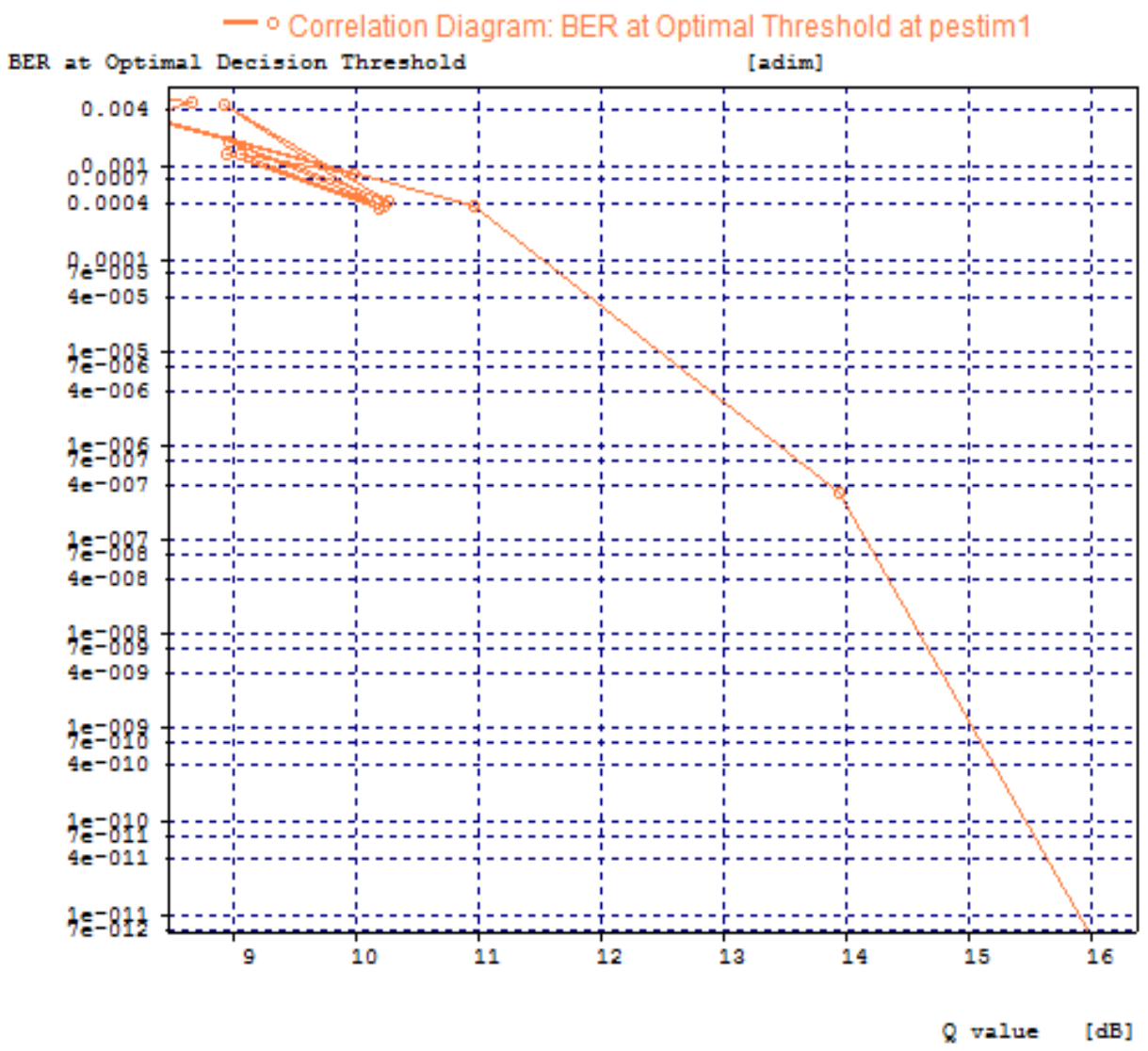

Fig (10) Computed BER with electrical generator for two transmitting antennas and one receiving antenna

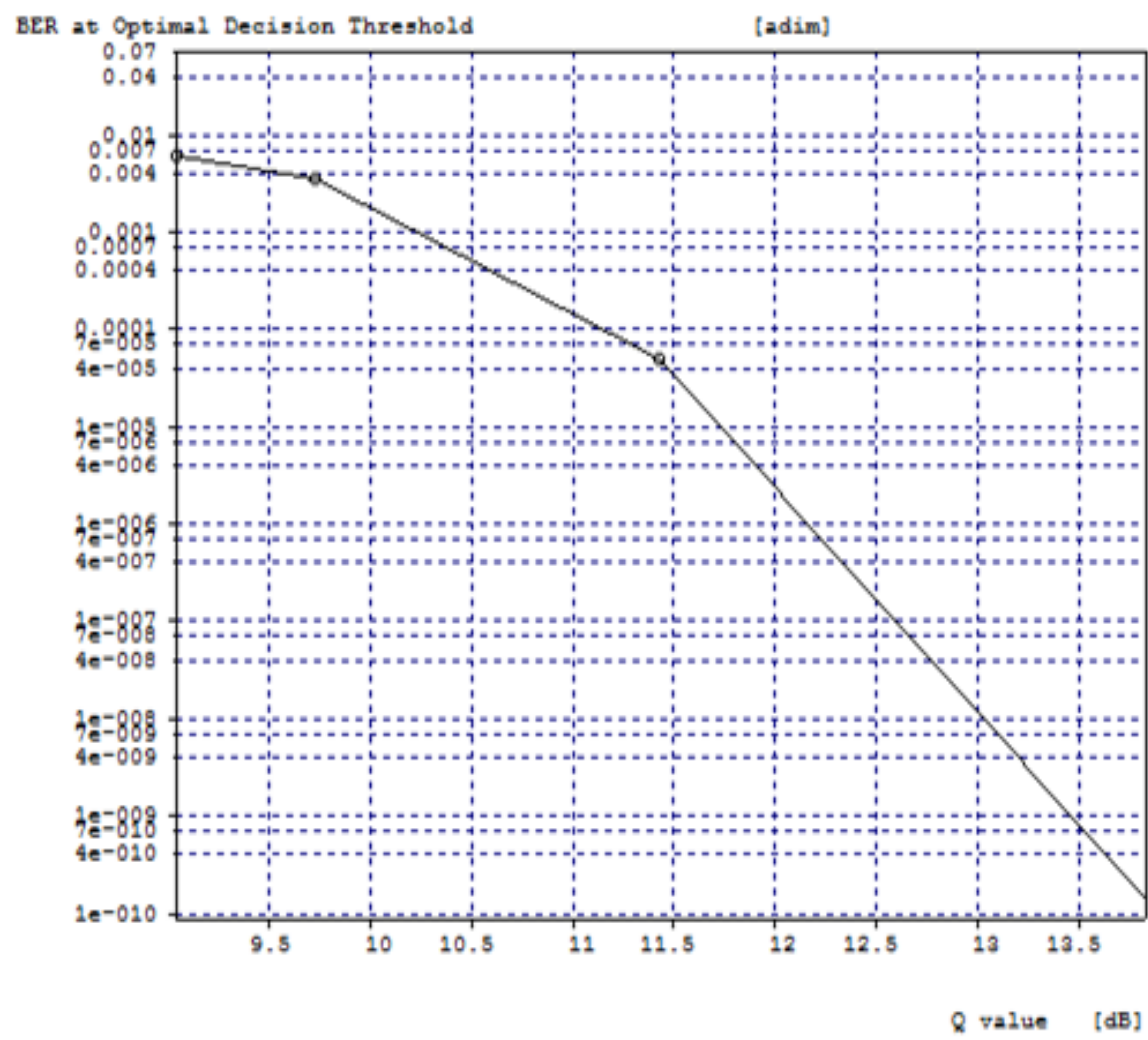

Fig (11) Computed BER with electrical generator for two transmitting antennas and two receiving antennas 


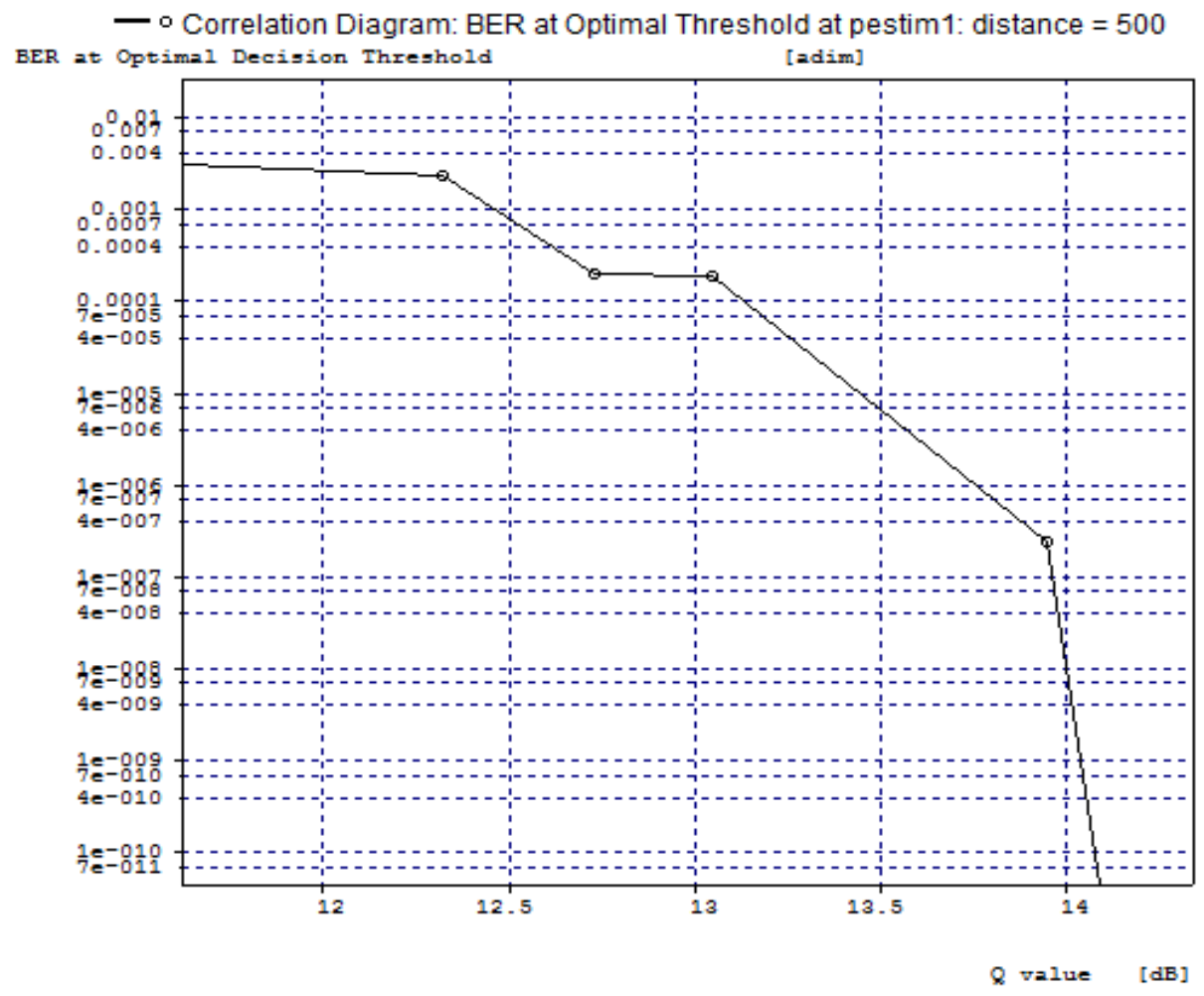

Fig (12) Computed BER with all optical system using MZI

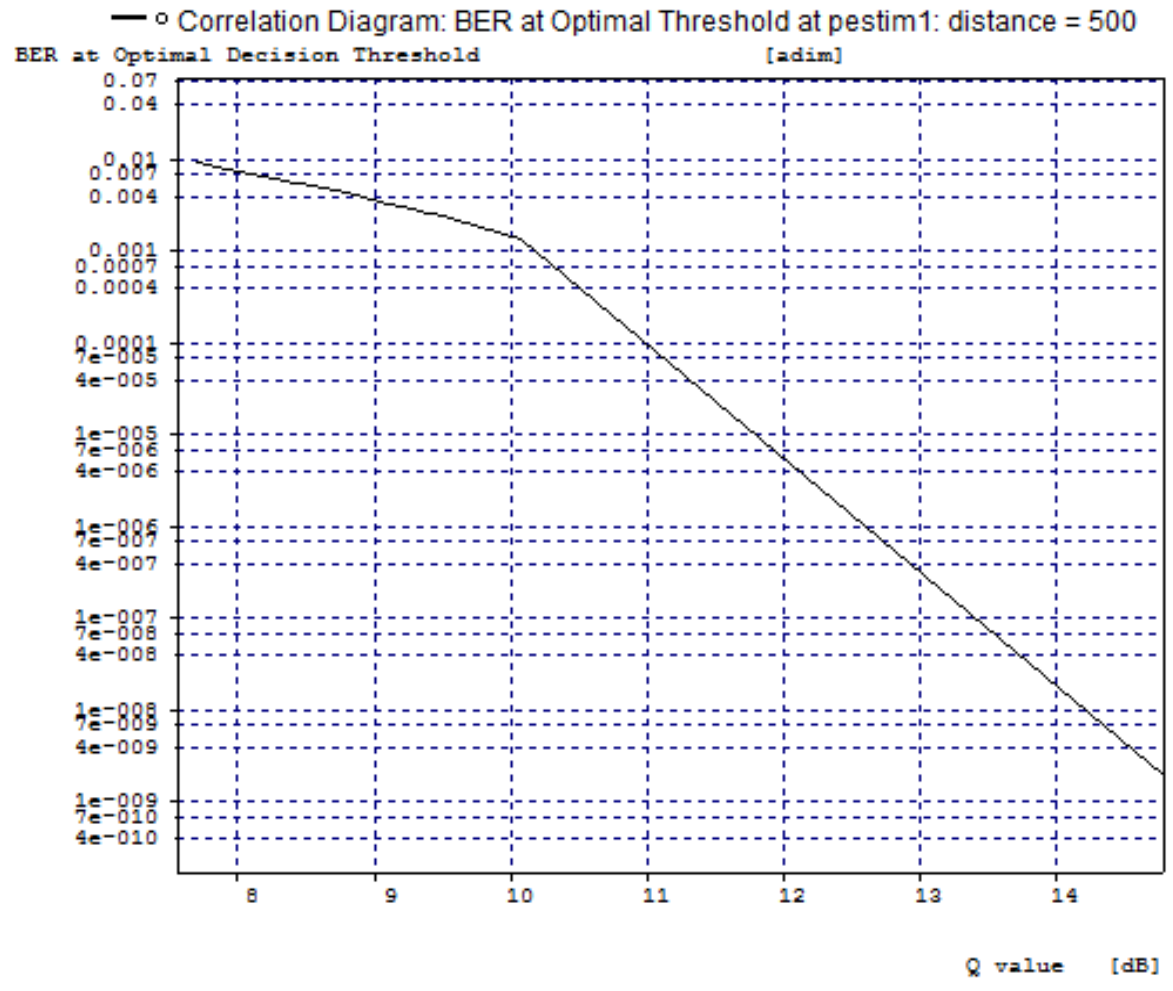

Fig (13) Computed BER with all optical system using EDFRL

\section{Conclusions}

This essay has discussed the reasons for designing an all optical wireless communication system based optical nonlinear signals generated by optical components to reduce overall system complexity. The main goal of the current work was to investigate the ability of using multiple inputs multiple outputs channels to improve the system performance. The important advantage of adopting optical generators instead of electrical 
generators is that the receiver does not need an additional beam steering which is necessary in optical wireless communications whereas aligned a narrow beam pointing back to destination point. The findings reported here that the overall system performance for all optical system has been equivalent to the optical wireless communication system using electrical components with gaining less system complexity due to using all optical components.

\section{References:}

[1] A, Mohamed Abaza, Manav R. Bhatnagar and Virander Kumar Jain, "An Investigation of Performance and Diversity Property of Optical Space Shift Keying Based FSO-MIMO System IEEE Transactions on Communications, DOI 10.1109/TCOMM.2018.2818702, p.p. 1-16, 2018.

[2] X, Xian Wang and Chi Yeh Hsu, "Design and Implementation of Mobile Free Space Optical Communication System", IEEE Conference of Avionics, Fiber Optic and Photonics Technology, p.p. 37-38, 2008.

[3] M. Premaratne and E-C Zheng, "Orthogonal spacetime block codes for free-space IM-DD optical links", IEEE Electronic Letter, Vol. 43, Issu:15, p.p. 822-823, 2007.

[4] S. I. Al-Nassar, H. Hatem and J. Shehab, "Design and Implementation of Infrared (IR) Communication

System", Diyala Journal of Engineering Sciences, Vol.

11, No. 3, September 2018, pages 29-33.

[5] E, Robert Schober and Ranjan K. Mallik, "On SpaceTime Coding for Free-Space Optical Systems", IEEE Transactions on Communications, Vol. 58, No. 1, p.p. 5862, 2010.

[6] E, Robert Schober and Ranjan K. Mallik, "Performance Analysis of MIMO Free-Space Optical Systems in Gamma-Gamma Fading", IEEE Transactions on Communications, Vol. 57, No. 11, p.p.3415-3425, 2009.

[7] S M. Alamouti, "A Simple Transmit Diversity Technique for Wireless Communications", IEEE Journal on Select Areas in Communications, Vol. 16, No. 8, p.p. 1451-1458, 1988.

[8] H Y and TomoakiOhtsuki, "Atmospheric Optical Subcarrier Modulation Systems using Space-Time Block Code", IEEE Global Telecommunication Conference, Vol. 6, p.p.3326-3330, 2003.

[9] M TaghiDabiri, Mohammad Javad Saber and Seyed Mohammad SajadSadough, "On the Performance of Multiplexing FSO MIMO Links in Log-Normal Fading With Pointing Errors", J. OPT. COMMUN. NETW, Vol. 9, No. 11, NOVEMBER 2017.

[10] S. Donati and C. R. Mirasso, "Introduction to the Feature Section on Optical Chaos and Applications to
Cryptography", IEEE Quantum of Electronics Vol. 38, No. 9, p.p. 1138-1140, 2002.

[11] S. Habeeb, "Effect of Weather Relation on Optical Interferometer Modulator", Diyala Journal of Engineering Sciences, Vol. 08, No. 02, pp. 151-162, June 2015.

[12] X. Lin, C. Yu and Q. Zhang, "Generator of Optical Chaotic Sequences", IEEE Electronic Letters, Vol. 43, No. 21, p.p. 1195, 2007.

[13] L. G. Luo, P. L. Chu, and H. F. Liu, "1 GHz optical communication system using chaos in erbium-doped fiber lasers," IEEE Photon. Technol. Lett., vol. 12, pp. 269271, Mar. 2000.

[14] S. Kim, B. Lee, and D. H. Kim, "Experiments on chaos synchronization in two separate erbium-doped fiber lasers," IEEE Photon. Technol. Lett., vol. 13, pp. 290292, Apr. 2001.

[15] R Rovatti, GianlucaSetti, and Gianluca Mazzini, "Chaotic Complex Spreading Sequences for Asynchronous DS-CDMA-Part II: Some Theoretical Performance Bounds", IEEE Transactions on Circuits and Systems-1, Fundamental Theory and Application, Vol. 45, No. 4, p.p. 496-506, 1998.

[16] G Mazzini, Riccardo Rovatti, and Gianluca Sett, "Chaos-Based Asynchronous DS CDMA Systems and Enhanced Rake Receivers: Measuring the Improvementsi", IEEE Transactions on Circuits and Systems-1, Fundamental Theory and Application, Vol. 48, No. 12, p.p. 1445-1453, 2001.

[17] S. Z. Ali, M.K. Islam and M. Zafrullah, "Generation of higher degree chaos by controlling harmonics of the modulating signal in EDFRL", Elsevier, p.p.1903-1909, 2011.

[18] S. Z. Ali, M.K. Islam and M. Zafrullah, "Comparative analysis of chaotic properties of optical chaos generators”, Elsevier, p.p.850-955, 2012.

[19] S. Z. Ali, "Generation and control of chaos for secure optical communication using EDFRL", PhD Thesis, Chapter two, 2011.

[20] L Faisal Abdulameer, HalaFadhil, "Performance Analysis of FSO under Turbulent Channel Using OSTBC", Al-Nahrain Journal for Engineering science, Vol. 2, No. 3, p.p. 344-349, 2018. 